\section{Treatment of acute colonic diverticular bleeding in high risk patients, using an over-the-scope clip: a case series}
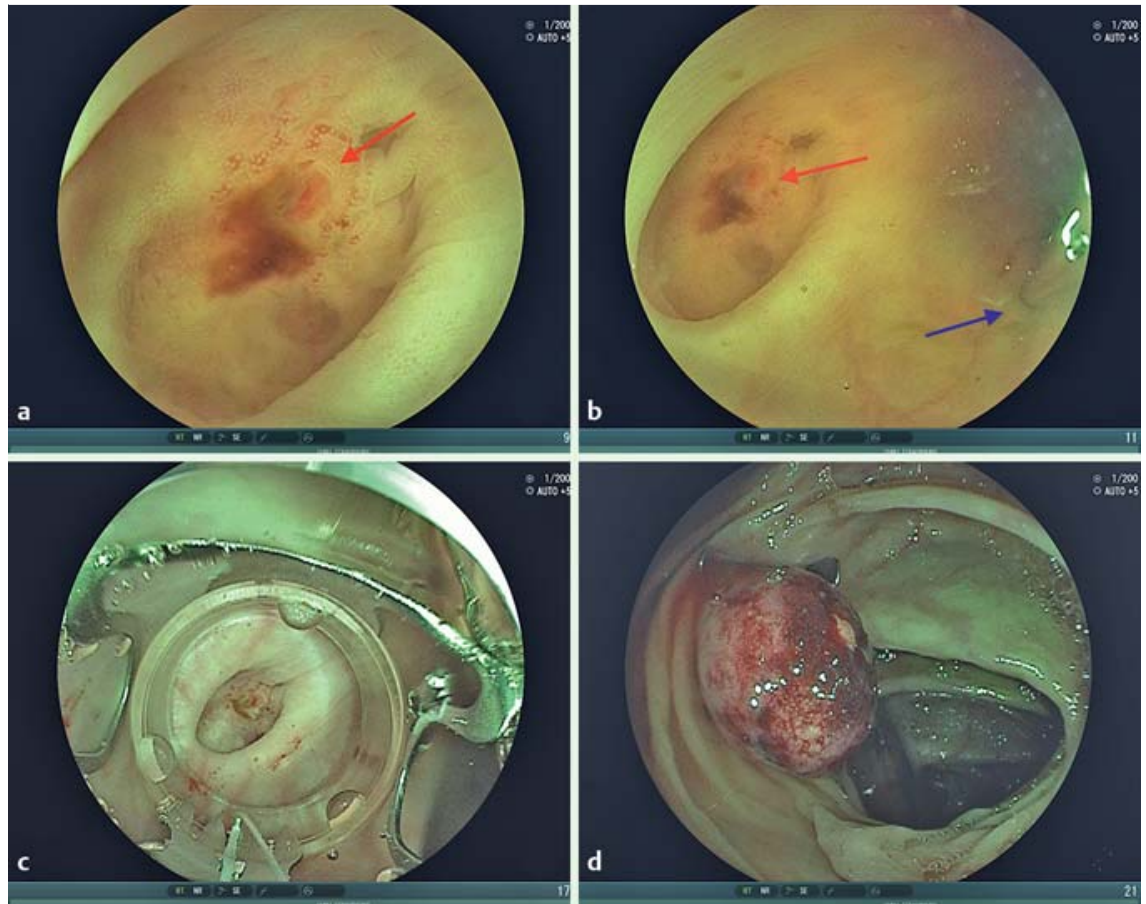

Fig. 1 Endoscopic application of the over-the-scope clip (OTSC) for treatment of acute colonic diverticular bleeding. a A diverticulum with a visible vessel $(0.3 \mathrm{~mm}$, red arrow) was detected in the sigmoid colon. $\mathbf{b}$ The site was marked with a standard hemoclip (blue arrow). $\mathbf{c}$ In a second step, the target diverticulum was inverted into the cap of the OTSC by suction. $\mathbf{d}$ The OTSC was then released.

The prevalence of diverticulosis has increased in industrialized countries in recent years and the condition is reported in up to $60 \%$ of patients older than 70 years $[1,2]$. Diverticular disease is one of the most common causes of lower gastrointestinal bleeding and accounts for $30 \%-$ $40 \%$ of all such bleeds [3,4]. Many diverticular bleeding episodes resolve spontaneously. However, according to the literature about $50 \%$ of patients require blood transfusion and 18\%-53\% need emergency surgery [2]. Patients undergoing anticoagulation treatment or presenting with severe hemorrhage are at high risk of both morbidity and mortality.

The treatment of diverticular hemorrhage remains controversial. If endoscopy can identify the bleeding diverticulum and disrupted inner vessel, therapies such as epinephrine injection, electrocautery, rubber band ligation, or hemoclip placement may be used $[3,4]$.

In this case series we describe six patients with a high operative and rebleeding risk who received an over-the-scope clip ([OTSC]; $17.5 \mathrm{~mm}$ or $21 \mathrm{~mm}$ ) for the treatment of colonic diverticular bleeding with an identifiable bleeding source. Four of the six patients in our series were receiving anticoagulation or antiplatelet therapy. The fifth individual was morbidly obese, with a body mass index of $38 \mathrm{~kg} / \mathrm{m}^{2}$. The sixth patient presented with hemorrhagic shock.

For OTSC placement, a standard adult colonoscope (e.g. EC590Z M-type; Fujifilm, Tokyo, Japan) was used. In patients with an actively bleeding diverticulum, or with bleeding stigmata such as a visible vessel or sentinel clot within the diverticulum, the diverticulum was first marked cranially with a standard clip ( $\bullet$ Fig. 1 a, b; - Video 1). The endoscope was then withdrawn and the OTSC was mounted on its distal end. The endoscope was reinserted, and the lesion was centered within the transparent cap of the OTSC. Suction was carefully applied in order to allow an inversion of the diverticulum into the cap

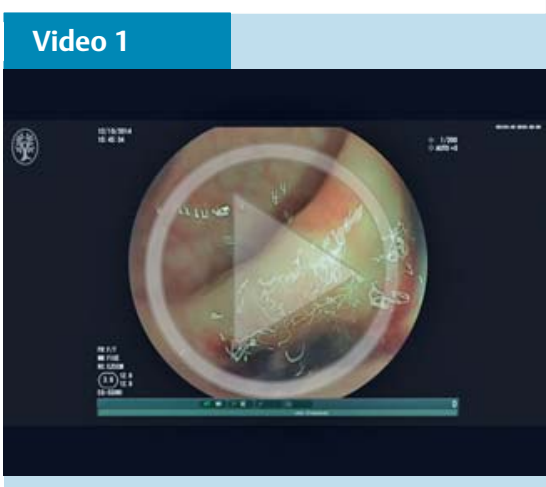

Detection of acute colonic diverticular bleeding in high risk patients, and treatment with an over-the-scope clip.

together with the surrounding normal colonic wall. The OTSC was then slowly released from the transparent application cylinder ( $\bullet$ Fig. 1 c, d). Correct application of the OTSC and primary hemostasis was achieved in all patients.

- Table 1 gives an overview of patient and treatment characteristics, and results. In four patients the OTSC remained the only endoscopic treatment, and all these patients had an uneventful further clinical course. One patient presenting with diverticular bleeding in the ascending colon (patient 2) developed severe rebleeding at the diverticular site. A second OTSC was successfully applied on the central tissue mound as well as three standard hemoclips. The patient remained asymptomatic and had an uneventful course. The second case of recurrent bleeding occurred 13 days after OTSC treatment in a patient with a mechanical aortic valve who was receiving therapeutic anticoagulation (patient 3). Recurrent bleeding in the sigmoid colon was treated using fibrin glue injection into the central tissue mound plus application of a standard hemoclip.

The rebleeding in two of these high risk patients illustrates both the advantage and the limitations of the OTSC. The sawtooth-like configuration of the macroclip permits sufficient perfusion of the centrally captured tissue and prevents necrosis in a similar way to a surgical stapler. The sharp teeth of the OTSC t-type clip used allow secure anchoring within the normal tissue surrounding the neck of 


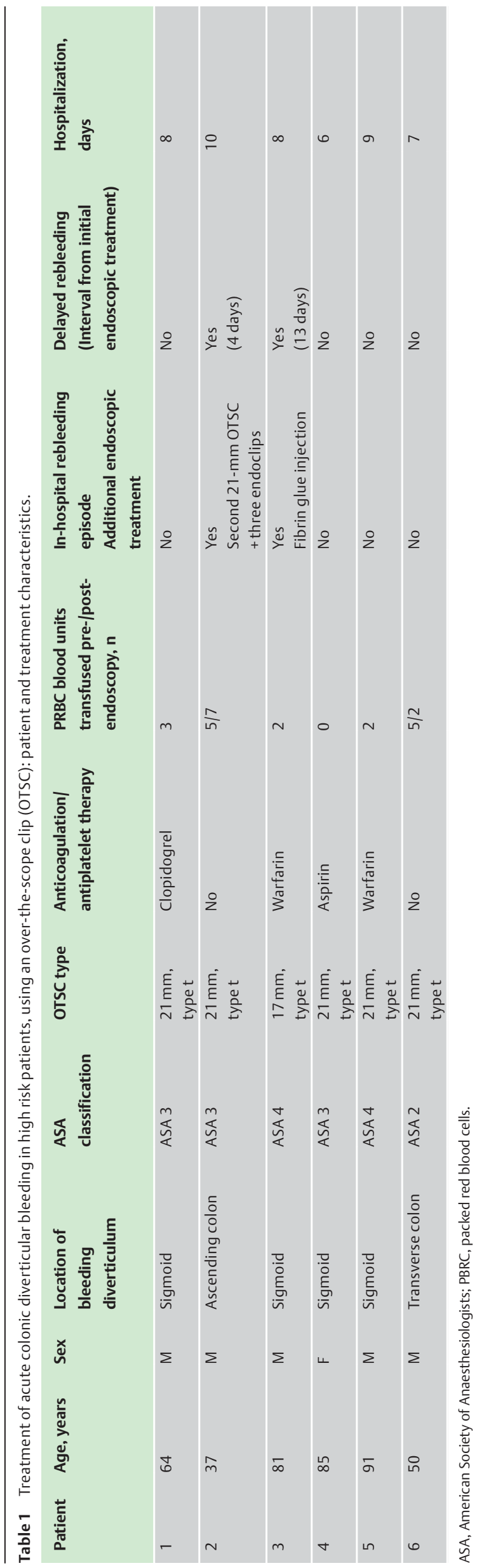

the diverticulum. Both characteristics may be advantageous compared with rubber band ligation.

The findings from our case series presented here confirm the results recently published by Probst et al. for a case of diverticular bleeding in a patient undergoing anticoagulation therapy [5].

To our knowledge, this is the largest case series published on the use of the OTSC system for the treatment of diverticular bleeding in high risk situations. We believe that OTSC placement is a safe and effective new option for patients in whom the bleeding diverticulum can be identified endoscopically.

Endoscopy_UCTN_Code_TTT_1AQ_2AZ

\section{Competing interests: None}

Edris Wedi ${ }^{1,2}$, Daniel von Renteln ${ }^{3}$, Carlo Jung ${ }^{1,2}$, Irina Tchoumak ${ }^{1}$, Victor Roth ${ }^{1}$, Susana Gonzales ${ }^{4}$, Joel Leroy ${ }^{5}$, Juergen Hochberger ${ }^{1,6}$

${ }^{1}$ Department of Gastroenterology, Nouvel Hôpital Civil, University Hospitals, Strasbourg, France

2 Department of Gastroenterology and GI Oncology, Goettingen University Hospital, Goettingen, Germany

${ }^{3}$ Division of Gastroenterology, Centre Hospitalier de l'Université de Montréal (CHUM), Research Centre CHUM (CRCHUM) Montreal, Quebec, Canada

${ }^{4}$ Division of Gastroenterology, NYU Langone Medical Center, New York, NY, USA

${ }^{5}$ Department of Surgery, Nouvel Hôpital Civil and IHU Strasbourg, University Hospitals, Strasbourg, France

${ }^{6}$ Division of Gastroenterology, Vivantes Klinikum in Friedrichshain, Teaching Hospital of Humboldt University, Charité, Berlin, Germany

\section{Acknowledgments}

This case series was presented as a poster at the Annual Meeting of the German Society of Endoscopy (DEGBV) in Mannheim, Germany, 17-19 March 2016.

\section{References}

1 Hughes LE. Postmortem survey of diverticular disease of the colon. II. The muscular abnormality of the sigmoid colon. Gut 1969; 10: $344-351$

2 Buttenschoen $K$, Buttenschoen DC, Odermath $R$ et al. Diverticular disease-associated hemorrhage in the elderly. Langenbeck's archives of surgery/Deutsche Gesellschaft fur Chirurgie 2001; 386: 8-16 
3 Gayer C, Chino A, Lucas C et al. Acute lower gastrointestinal bleeding in 1,112 patients admitted to an urban emergency medical center. Surgery 2009; 146: 600-606; discussion 606-607

4 Ghassemi KA, Jensen DM. Lower GI bleeding: epidemiology and management. Curr Gastroenterol Rep 2013; 15: 333

5 Probst A, Braun G, Goelder S et al. Endoscopic treatment of colonic diverticular bleeding using an over-the-scope clip. Endoscopy 2016; 48 (Suppl. 01): E160
Bibliography

Dol http://dx.doi.org/

10.1055/s-0042-118168

Endoscopy 2016; 48: E383-E385

(c) Georg Thieme Verlag KG

Stuttgart · New York

ISSN 0013-726X

\section{Corresponding author}

\section{Edris Wedi, MD}

Department of Gastroenterology and GI Oncology Universitaetsmedizin Goettingen

Georg-August-Universitaet

D-37099 Goettingen

Germany

Fax: +49-512-1901241

edriswedi@gmail.com 